\title{
Atrial Electro-anatomic Mapping with a Novel Noncontact Approach
}

\author{
Meng, S. ${ }^{1}$, Zhao, J. ${ }^{1}$, LeGrice, I. ${ }^{1,2}$, Lever, N.. ${ }^{1,3}$, Sands, G. ${ }^{1}$, Bear, L. ${ }^{4}$, Gillis, A. ${ }^{5}$, Smaill, B. ${ }^{1,2}$ \\ ${ }^{1}$ Auckland Bioengineering Institute, ${ }^{2}$ Department of Physiology, University of Auckland and \\ ${ }^{3}$ Auckland City Hospital, Auckland, New Zealand, ${ }^{4}$ IHU-LIRYC, Fondation Bordeaux Université, \\ Bordeaux, France, ${ }^{5}$ Libin Cardiovascular Institute of Alberta, University of Calgary, Canada
}

\begin{abstract}
Multi-electrode basket catheters are used for $3 D$ mapping of complex focal arrhythmias in real-time, but full contact is hard to achieve for all electrodes in the atria. We have developed a novel inverse mapping approach, which enables endocardial potentials to be recovered from noncontact recordings and report on its efficacy.

Intra-atrial electrograms were acquired with 64 channel Constellation ${ }^{\mathrm{TM}}$ catheters in 4 anesthetized sheep in sinus rhythm (SR), atrial pacing (AP) and atrial fibrillation $(A F)$, with catheters of different dimension at various locations in right and/or left atria (RA, LA). Atrial endocardial potentials were recovered from noncontact recordings using finite-element and meshless inverse methods.

Inverse mapping reproduced contact electrograms, recorded separately at distributed sites, with excellent accuracy in SR and AP [CC 0.8 \pm 0.18 , time delay $1 \pm 3 \mathrm{~ms}$, relative $R M S$ error $0.53 \pm 0.17, n=96]$. Moreover, repeatable activation time maps were obtained in SR and $A P$. Focal activity was identified in $A F$ and precise noncontact region-of-interest mapping was also demonstrated.
\end{abstract}

\section{Introduction}

Atrial fibrillation (AF) is the most common heart rhythm disturbance and percutaneous catheter ablation is widely used to isolate the pulmonary veins and reverse paroxysmal AF [1]. This approach has been much less successful for patients with persistent $\mathrm{AF}(\mathrm{PeAF})$ [1] and real-time mapping of 3D electrical activity is needed to identify ablation targets in this complex nonstationary arrhythmia [2]

Multi-electrode basket catheters have been used to map of complex focal arrhythmias in real-time, but it is hard to achieve uniform contact between electrodes and the inner surface of the atrial surface. Inverse methods in which surface potentials are reconstructed from noncontact recordings may provide a better alternative. This approach is employed with the EnSITE (St Jude Medical) multi-electrode balloon catheter, but the electrograms recovered in PeAF are subject to artifact, because the electrodes are too distant from the atrial wall [3]. Here, we describe novel inverse mapping methods that can be used with open multi-electrode basket catheters when some or all electrodes are not in contact with the atrial wall.

\section{Mathematical background}

Noncontact intracardiac potential mapping seeks to recover electrograms on the endocardial surface $\Gamma_{\mathrm{e}}$ of a cardiac chamber $\Omega$ from recordings made with a multielectrode catheter inside it. Intracardiac basket catheters do not obstruct blood flow and it is assumed that $\sigma$, the conductivity of $\Omega$, is uniform and isotropic. Because there are no electrical sources in $\Omega$, the potential $\Phi$ within the domain obeys Laplace's equation

$$
\nabla^{2} \Phi=0
$$

Provided that appropriate boundary conditions are specified on $\Gamma_{\mathrm{e}}$, the forward problem (1) can be solved uniquely and $\Phi$ can be determined on the arbitrary surface $\Gamma_{\mathrm{c}}$ that bounds the catheter. The corresponding inverse problem can also be solved if both $\Phi$ and $\partial \Phi / \partial \mathrm{n}$ are specified on $\Gamma_{\mathrm{c}}$, where $\mathbf{n}$ indicates the surface normal direction [4]. However, the inverse problem is inherently underdetermined and solutions are not unique. Furthermore, boundary conditions are not fully specified with a basket catheter, because current can flow through it and therefore $\partial \Phi / \partial \mathrm{n} \neq 0$ at $\Gamma_{\mathrm{c}}$. We have developed novel methods that enable inverse potential mapping in this setting. These include i) accurate interpolation of instantaneous potentials on $\Gamma_{\mathrm{c}}$ from electrograms sampled at restricted numbers of electrodes and ii) estimation of $\partial \Phi / \partial \mathrm{n}$ across $\Gamma_{\mathrm{c}}$.

\section{Methods}

\subsection{Experimental Study}


Noncontact atrial mapping was performed in 4 anaesthetized, ventilated sheep (30-50 kg) with pacinginduced atrial dilatation. Constellation ${ }^{\mathrm{TM}}$ (Boston Scientific) 64 channel catheters $(38,64$ and $60 \mathrm{~mm})$ were introduced into the right atrium (RA) and/or the left atrium (LA). Atrial electrograms on all electrodes were digitized $3 \mathrm{kHz}$ (12 bit resolution; bandwidth $0.5 \mathrm{~Hz}-$ $1.5 \mathrm{kHz}$ ) and recorded with a UnEMap multi-channel acquisition system, (Auckland Uniservices) in sinus rhythm, during RA pacing and in AF with catheters of different dimensions at a range of different atrial locations. Biplane ciné fluoroscopic images of the atria were acquired (PHILIPS BV Pulsera) with ECG superimposed, before or after each electrical recording. The atria were imaged in vivo in a 3T MRI Scanner (Siemens Magnetom Skyra) using gadolinium contrast-enhancement.

\subsection{D reconstruction}

Atrial chamber geometry was segmented and reconstructed in 3D from the MRI image volumes. Because electrograms were not acquired in the atrial appendage, we truncated the surfaces used for inverse mapping at the junction of atria and atrial appendages. The 3D locations of electrodes and other fiducial markers were estimated from biplane ciné-images using wellestablished projection methods [5]. Electrode locations were reconstructed at the onset of atrial activation (Figure 1A). Electrode positions along each catheter spline were smoothed with least squares spline functions to reduce reconstruction error and a surface $\Gamma_{\mathrm{c}}$ that bounded the electrodes was then fitted to these data. Finally, reconstructed catheters and fiducial markers were registered with $3 \mathrm{D}$ atrial geometry.

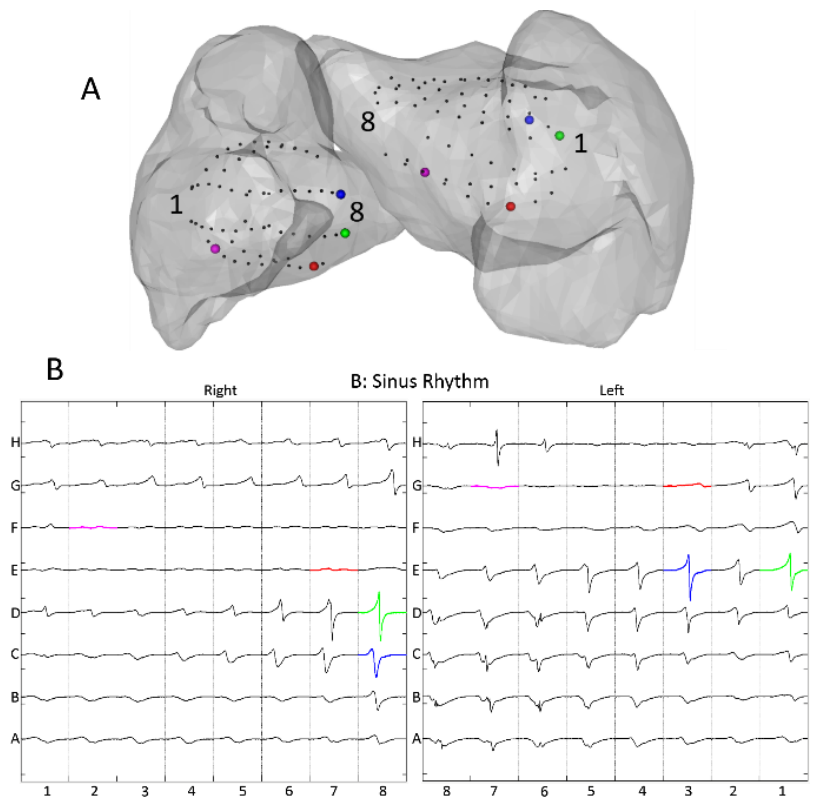

Figure 1: (A) Bi-plane reconstructed catheters registered in segmented RA and LA. (B) Intracavity electrograms recorded in sinus rhythm with $48 \mathrm{~mm}$ and $60 \mathrm{~mm}$ Constellation catheters in RA and LA, respectively.

\subsection{Boundary conditions}

It is necessary to interpolate potentials across the virtual surface $\Gamma_{\mathrm{c}}$ for better determined inverse solutions with finite element and boundary element methods. The distribution of electrodes on the Constellation catheter is too sparse to reconstruct the rapid spatio-temporal variation generated by the atrial activation wavefront fully, using standard spatial interpolation procedures [6]. We demonstrate here that spatial interpolation in the frequency domain recovers propagating wavefronts with much less artifact, because amplitude and phase spectra are distributed uniformly, whereas instantaneous potentials are not. Magnitude and phase spectra were determined for recorded electrograms and estimated at 3D points between electrodes by spatial interpolation of harmonic components. Corresponding electrograms were then reconstructed using the inverse Fourier transform.

Interpolated potentials were used to estimate $\partial \Phi / \partial \mathrm{n}$ on $\Gamma_{\mathrm{c}}$ at successive time steps throughout atrial activation. At each instant, $\Phi$ was estimated in the volume bounded by $\Gamma_{\mathrm{c}}$ by solving the forward problem within this subdomain. Finite difference methods were used with Dirichlet boundary conditions specified by the interpolated potentials on $\Gamma_{\mathrm{c}}$. Potential gradients normal to $\Gamma_{\mathrm{c}}$ were then approximated from the potential field adjacent to that virtual surface.

\subsection{Preprocessing and visualization}

Most data processing steps above were performed in the Matlab programming language (The Mathworks). A purpose-coded GUI was developed for signal processing, data comparison and visualization, also in Matlab.

\subsection{Inverse solutions}

Inverse solutions for the specified boundaries and boundary conditions were obtained using the finiteelement method with the SCIRun software platform (Center for Integrative Biomedical Computing, University of Utah). The truncated atrial chamber was represented by 402 triangular mesh points and the virtual 3D surface bounding electrodes on the Constellation catheter was represented by 6720 mesh points. Tikhonov regularization was employed and the L-curve technique was used to estimate the regularization parameter [7]. Results were compared with corresponding solutions obtained using meshless methods with purpose-developed 
code. Activation times for electrograms reconstructed on the atrial surface were determined initially from the maximum negative derivative and were smoothed using the time-delay method [8].

\section{Results}

\subsection{Recorded electrograms}

In Figure 1B, we present typical electrograms recorded in the RA and LA (48 and $60 \mathrm{~mm}$ catheters, respectively) in sinus rhythm. As expected, electrodes close to the atrial wall record large amplitude electrograms with rapid temporal variation, whereas electrograms recorded further from the atrial surface are more slowly varying and have low amplitude. Finally electrograms toward the center of the cavity and closest to the inlet valves appear to have very little signal content. It is difficult to interpret the low amplitude signals or to relate the recorded electrograms to activation spread to activation spread on the atrial surface.

\subsection{Interpolated electrograms}

The advantages of frequency domain potential interpolation are shown in Figure 2. Radial basis function interpolation does not reproduce the uniform spread of an activation waveform across $\Gamma_{\mathrm{c}}$ (Figure 2A), because the distribution of electrodes along splines is relatively sparse and even more so between splines. In contrast this spread is recovered more faithfully and more efficiently with spatial interpolation in the frequency domain (Figure 2B), because temporal information is incorporated in the interpolation process. This regularizes interpolation between splines, but also reduces interpolation artifact along splines.
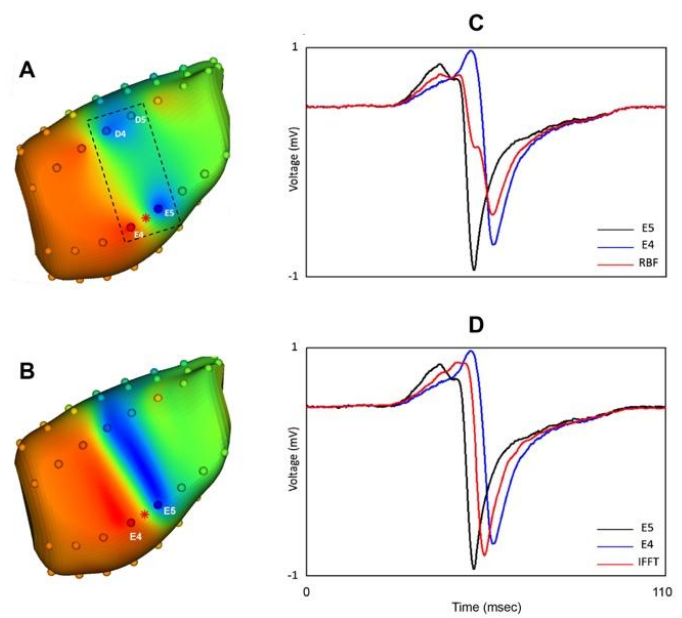

Figure 2: Spatial interpolation of potentials. Left-hand panel: Instantaneous potentials interpolated on $\Gamma_{\mathrm{c}}$ using
(A) radial basis function interpolation and (B) frequency domain interpolation. Righthand panel: Electrograms interpolated midway between adjacent electrodes on a spline with (C) radial basis function interpolation and (D) frequency domain interpolation.

In Figure 2C, an electrogram reconstructed midway between two electrodes by sequential radial basis function interpolation does not recover the rapid potential variation that occurs during depolarization, because the spatial sampling is too coarse. On the other hand, corresponding frequency domain interpolation (Figure 2D) captures smooth wavefront progression. This interpolation procedure would be optimized by the development of intracardiac catheter arrays with a more uniform spatial distribution of electrodes than is provided by the Constellation catheter.

\subsection{Inverse electrical mapping}

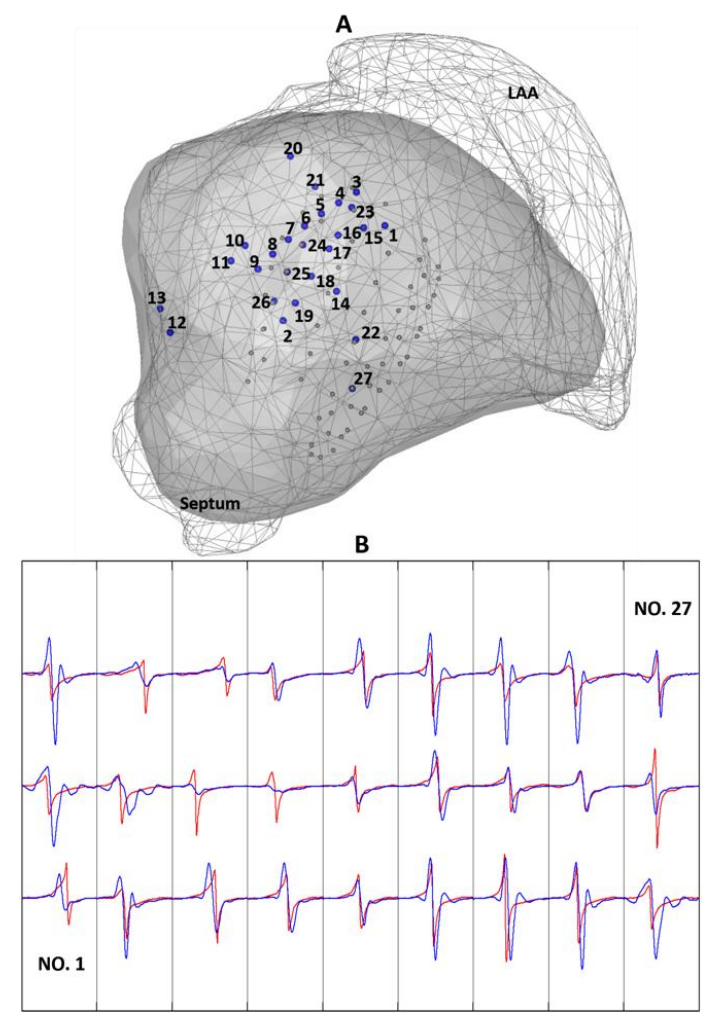

Figure 3: Comparison electrograms recorded on LA surface with electrograms reconstructed at the same points from noncontact recordings using inverse potential mapping. (A) Location of contact recordings and basket catheter electrodes (open circles). (B) Recorded (red) and reconstructed electrograms (blue) during sinus rhythm. 
The efficacy of inverse potential mapping was assessed by comparing contact electrograms acquired at distributed atrial sites in sinus rhythm and during atrial pacing with electrograms reconstructed at these sites from noncontact recordings. Typical LA electrograms recorded and reconstructed during atrial pacing are presented in Figure 3. Electrogram shape is recovered relatively well and timing is matched closely. These findings were replicated within and across animals, and the following mean values for multiple noncontact recordings were : correlation coefficient $0.8 \pm 0.18$, time delay $1 \pm 3 \mathrm{~ms}$, relative RMS error $0.53 \pm 0.17, n=96$. As indicated by the relative RMS error, the amplitude of recovered electrograms was variable and this was greatest where electrodes were distant from the atrial surface in regions of low signal strength. However, electrogram morphology and timing were recovered faithfully. Reflecting this, global activation maps were physiologically consistent and repeatable. As seen in Figure 4A, activation spread first from the superior RA adjacent to the junction with the superior vena cava in sinus rhythm and total activation time was $\sim 60 \mathrm{msec}$. With atrial pacing, activation spread from the pacing site and was completed in $\sim 85 \mathrm{msec}$. Activation time maps were also relatively invariant with catheters of a range of dimensions and intracavity locations (see Figure 4B).
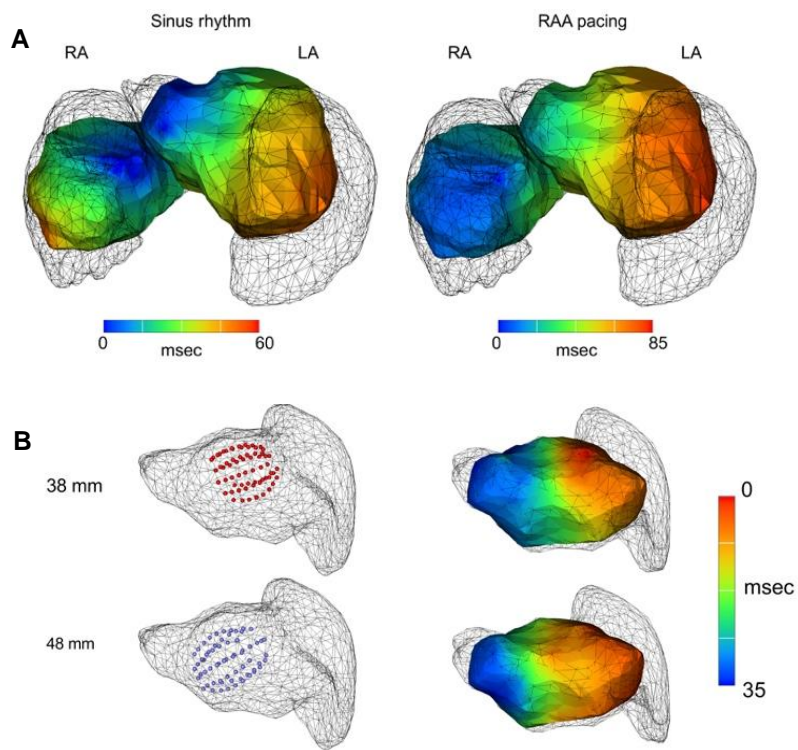

Figure 4: Atrial activation time maps derived with inverse methods. (A) Activation maps in sinus rhythm and atrial pacing obtained from simultaneous recordings with 48 and $60 \mathrm{~mm}$ Constellation catheters in RA and LA, respectively. (B) LA activation maps in atrial pacing obtained from recordings with 38 and $48 \mathrm{~mm}$ Constellation catheters.
Higher resolution noncontact electrical maps were recovered from collapsed $38 \mathrm{~mm}$ catheters close to specific regions of interest. Activation spread was also mapped (though not validated) in AF. Very similar inverse mapping results were obtained using meshless methods. This approach is computationally more efficient than mesh-based finite element and boundary element methods enabling near real-time inverse mapping.

\section{Conclusions}

We have demonstrated the feasibility of global and region-of-interest inverse potential mapping in the atria using noncontact basket catheters .

\section{Acknowledgements}

We acknowledge the assistance provided in this study by St Jude (Australia) Pty, Johnson \& Johnson (NZ) Ltd and Metronics Inc.

\section{References}

1. Brooks, A.G., et al., Outcomes of long-standing persistent atrial fibrillation ablation: A systematic review. Heart Rhythm, 2010. 7(6): p. 835-846.

2. Chao, T.-F., et al., Clinical outcome of catheter ablation in patients with nonparoxysmal atrial fibrillation: Results of 3-Year Follow-Up. Circulation: Arrhythmia and Electrophysiology, 2012. 5(3): p. 514-520.

3. Haissaguerre, M., et al., Driver domains in persistent atrial fibrillation. Circulation 2014.130(7):p. 530-538.

4. Barr, R. C. and M. Spach. Inverse calculation of QRS-T epicardial potentials from body surface potential distributions for normal and ectopic beats in the intact dog. Circulation Research 1978: 42(5): p. 661-675.

5. Dumay, A., et al., Determination of optimal angiographic viewing angles: Basic principles and evaluation study. IEEE Transactions on Medical Imaging, 1994. 13(1): p. 13-24.

6. $\mathrm{Ni}, \mathrm{Q}$., et al., Three-dimensional activation mapping in ventricular muscle: interpolation and approximation of activation times. Ann Biomed Eng, 1999. 27(5): p. 617-26

7. Tikhonov, A, Arsenin, V. Solution of ill-posed problems. John Wiley \& Sons, Washington, DC, 1977.

8. Walton, R. D., O. Bernus and R. Dubois (2013). A novel approach for deriving global activation maps from nonaveraged cardiac optical signals. 2013 35th Annual International Conference of the IEEE Engineering in Medicine and Biology Society (EMBC), IEEE.

Address for correspondence:

Shu Meng

Auckland Bioengineering Institute

E-mail:smen974@aucklanduni.ac.nz 